\title{
A Química no I Colóquio sobre a História e Desenvolvimento da Ciência em Portugal
} IV - A Análise Química *

Tomando como ponto de referência as Comunicações que no domínio da Química foram apresentadas no I Colóquio Sobre a História e Desenvolvimento da Ciência em Portugal, realizado na Academia das Ciências de Lisboa, de 15 a 19 de Abril de 1985, aqui nos referimos já à iatroquímica em Portugal, à actividade química na Universidade de Coimbra ao tempo da revolução química de Lavoisier, e à inestimável acção de químicos portugueses no campo da química dos alcaloides e polímeros. Para que se complete a nossa referência ao que naquele Colóquio se versou sobre a Química em Portugal, falaremos agora da Análise Química, tomando como referência a Comunicação do Professor da Faculdade de Ciências da Universidade do Porto, Dr. João Oliveira Cabral (ref. 1).

No prefácio da segunda edição da obra Précis d'Analyse chimique Qualitative escrevia Chancel, Professor da Faculdade de Ciências de Montpellier, em 1862: "os métodos analíticos atingiram a sua actual perfeição apenas há muito poucos anos; praticamente desconhecidos há um século, eles formam, hoje, um ramo especial da química" (ref. 2, pp. 3-4). Este ramo especial da química, como ramo sistematizado e autónomo, é a Química Analítica que se considera habitualmente ter nascido com Fresenius, entre 1841 e 1842 (ref. 1). Todavia, os esforços conducentes à sua sistematização vinham já de muito longe; são seus predecessores os processos de docimasia ligados a ensaios por via seca de minerais, conhecidos e praticados de há longa data. Porém, como o nota o mesmo Chancel, "a análise propriamente dita só se tornou verdadeiramente séria a partir do momento em que Lavoisier tendo formulado o seu grande princípio de que na natureza nada se perde, nada se cria deu aos químicos a lei da precisão que deve marcar seus trabalhos"' (ref, 2, pp. 4).

Em 1843, Barreswill e Sobrero publicavam o seu $A p$ pendice à Tous les Traités d'Analyse Chimique (ref. 3) que se propunha como objectivo primeiro ser uma recolha de todas as observaçðes sobre análise qualitativa e quantitativa depois da publicação, em 1832, do Traité Pratique d'Analyse Chimique da autoria de Henri Rosé. Este Apêndice aos Tratados de Análise Química abre com uma lista das principais obras publicadas em França, Inglaterra e Alemanha sobre o assunto. Elenco dos primeiros passos no domínio da análise química, as três mais antigas obras que nele se referem são as Prakt. Anleitung zur prüfenden und zerlegenden chemie de Götling (1802); Chemisches Laboratorium oder

- Part. I - A iatroquímica Portuguesa, Bol. Soc. Port. Quím., n. ${ }^{\circ}$ 21 (Série II), Set. 1985, pp. 61-63; Part. II - A Universidade de Coimbra e a Revolução Quimica de Lavoisier, idem, n. ${ }^{\circ} 22$ (Série II), Dez. 1985, pp. 47-50; Part. III - Alcalbides e Polimeros, idem, n. ${ }^{2} 2$ (Série II), Março 1986, pp. 37-39.
Anweisung zur Chemischen Analyse der Naturalien de John (1808); e Théorie des Atomes et des Équivalents Chimiques de Choron (1809). Desse elenco constam o tratado de Berzelius dedicado à análise dos corpos inorgânicos (1827), o Manual de Lietig para a análise das substâncias orgânicas (1838), e as consideraçðes gerais sobre a análise orgânica e suas aplicaçðes de Chevreul (1824).

Em Portugal, em 1814, o então Director do Laboratório Chimico da Universidade de Coimbra, Thomé Rodrigues Sobral escrevia longamente Sobre as difficuldades de uma boa Anályse (ref. 4), interessado na análise das "pedras e das terras, dos saes, dos metaes e suas minas, e das ágoas mineraes". Neste escrito se pode ler: "uma analyse qualquer, e muito especialmente uma anályse vegetal ou animal, he o trabalho mais difficil de toda a chimica"; ela "he como a pedra de toque que distingue $o$ chimico profundo, d'aquelle que $o$ não he. A parte analytica da chimica he a que exige conhecimentos mais extensos e mais profundos na Sciencia; applicaçס̃es mais prontas e felizes d'estes conhecimentos; he a que requer uma atenção mais escrupulosa a todos os phenómenos ainda os menos significantes em apparência, sendo todos muito importantes, quando são vistos a manejados por um chimico hábil e profundo" (ref. 4, pg. 252).

$\mathrm{E}$ de imediato define o objectivo de uma tal ciência: "a anályse chimica tem sempre este único problema que resolver: quantos e quaes são os princípios ou elementos, sejão próximos, sejão remotos, que compōem o corpo que se-analyza? Quaes são as proporçðes respectivas d'estes princípios, seja dos immediatos e ainda compostos; seja dos elementares, e solte os quaes os meios d'anályse actuaes ja nada podem?"' (ref. 4, pg. 252).

É reconhecida à relativa facilidade da análise dos minerais no domínio da qual confessa a sagacidade e exactidão dos trabalhos analíticos de Bergmann, e também a relativa facilidade da análise dos saes comı "corpos constando pela maior parte somente de três principios, a saber: um ácido, uma base e mais ou menos ágoa", que apesar de tudo se via a braços com situaçбes de grande disparidade, com casos em que a discrepância nos resultados da análise de um mesmo sal por diferentes cientistas ir, algumas vezes, além do duplo a respeito da proporção de alguns princípios.

No Laboratório Químico se procedia então a grande número de análises de metais e suas minas (ref. 5) e à análise simultânea e comparada de algumas espécies vegetais, nomeadamente certas quinas provenientes do

\footnotetext{
a Departamento de Quimica, Universidade de Coimbra 3000 Coimbra.
} 
Brasil e do Peru (ref. 6), não sem que aqueles que nelas trabalhavam estivessem bem cientes de "quanto uma boa anályse vegetal he árdua e difficil", e certos de que a análise vegetal estava ainda bem longe do ponto de perfeição que se desejava (ref. 4, pp. 262-263). Os ensaios analíticos que então levariam o Dr. Bernardino Antonio Gomes ao isolamento do cinchonino, no Laboratório Chimico da Casa da Moeda, em Lisboa, são disso prova clara, e a polémica que se gerou à volta da sua descoberta com o grupo de Coimbra, baseada fundamentalmente numa diferente interpretação dos resultados analíticos, testemunho vivo da reserva com que os resultados de muitas análises químicas efectuadas neste domínio eram recebidos (ref. 7). Praticamente no ponto-zero se encontravam então, na química portuguesa, as análises de águas e as análises toxicologicas. Numa referência às primeiras, diz o mesmo Rodrigues Sobral: "eu não conheço senâo duas anályses das nossas águas mineraes sulphureas ou thermaes, e outras duas d'águas marciaes. As duas primeiras, visto serem ambas relativas às Caldas da Rainha, me-offerecem quando as comparo a prova do que me propunha demonstrar - a difficuldade d'estas anályses pella differença de resultados - : a primeira que he do Dr. José Martins da Cunha Pessoa tem o inseparável merecimento de offerecer como o primeiro fructo da Sciencia Philosophica plantada na Universidade; e a extensão dos conhecimentos d'aquelle Sábio Clínico adquiridos no estudo da chimica Stahliana nos primeiros annos da Reforma da mesma Universidade; mas ella offerece necessariamente aquelles defeitos e imperfeiçōes, que o estado da Sciencia, a imperfeição ou a falta absoluta dos apparelhos, os poucos meios d'anályse que um chimico, ainda que hábil, tinha então, e que a mesma difficuldade da materia não permittião evitar. A segunda anályse he do Cel. Whitering, Philbsopho Inglez cujo trabalho analytico sobre estas aliás tão frequentadas Caldas, mandado fazer, ao que parece, pelo Ministério Inglez, he uma tácita reprehensão à nossa indifferença. A sua anályse differe em muito da que dera o Dr. Martins: mas elle mesmo reconhecendo as difficuldades de uma tal anályse, a importância do objecto, e a impossibilidade em que elle se-achava de o-desempenhar pela falta de instrumentos, convidou com a mais louvável candura os Philosophos Nacionaes a repetilla. A respeito das águas marciaes, de que abundamos, temos a Anályse das Águas Férreas de Torres-Novas Pátria do mesmo Sábio Médico Martins, e a que em Coimbra se-imprimio debaixo do nome de D. Francisco de Almeida (nota 8). Estes dois Escritos, posto que diffirão muito um do outro a alguns respeitos, não são contudo comparáveis por terem por objecto Águas Mineraes differentes.

"Tudo o que escreveram sobre Águas Mineraes um Fr. Christovão Boticário dos Padres Mariannos, e o Dr. Gago, Médico de Lisboa, não offerece mais do que os bons dezejos de seus AA. e as louváveis intençð̋es que tinham de serem úteis"' (ref. 4., pp. 258-259).

Fruto inegável da "Sciencia plantada na Universidade", a actividade mais significativa no domínio da análise química em Portugal, no âmbito do século XIX, desenvolve-se, em particular, por acção dos já referidos trabalhos que levaram Bernardino António Gomes (1768-1823) ao isolamento do cinchonino, e dos trabalhos de Luís da Silva Mousinho de Albuquerque (1792-1846), António Luís Ferreira Girão (1826-1876), Júlio Máximo de Oliveira Pimentel (1810-1885), Agostinho Vicente Lourenço (1822-1893), Joa- quim dos Santos e Silva (1842-1906) e António Joaquim Ferreira da Silva (1853-1923), entre outros.

"Estadista inteligente e de notável acção, escritor brilhante e consagrado", L. S. Mousinho de Albuquerque, na sua curta passagem pela Casa da Moeda de 1823 a 1826, distinguiu-se também como homem de ciência pela distinção com que seguiu, em Paris, os cursos de agricultura e de química de Vauglin e Dubois e, sobretudo, pela publicação do seu Curso Elementar de Física e de Química (ref. 9), e ainda pelo estudo que fez das águas de S. Miguel de que publicou um excelente relatorio nas suas Observaçôes sobre a Ilha de $S$. Miguel (ref. 10, pp. 19-20).

A. L. Ferreira Girão, Professor da Academia Politécnica do Porto desde 1859 , e nela proprietário da $\mathrm{Ca}$ deira de Química entre 1872 e 1876, "soube tirar proveito dos recursos que então tinha o laboratório de que era director para estudar e submetter à prova da experiência os melhores processos para determinar a existência do cobre, chumbo e zinco na água e nas bebidas fermentadas, e fazer o doseamento d'esses metaes nos mesmos líquidos"' (ref. 11). Sobre o assunto redigiu elucidativa monografia com o título: Ensaios Chimicos applicados à procura e doseamento dos compostos de chumbo, de cobre e de zinco nas águas potáveis e bebidas fermentadas; seguidos de várias experiências $e$ anályses.

J. M. Oliveira Pimentel (Conde de Vila Maior), primeiro Professor de química na Escola Politécnica de Lisboa, a quem se deve o primeiro tratado completo, em português, para a difusão dos estudos químicos, sob o título Lições de Química Geral e Suas Principais Applicaçōes (Lisboa, 1852), procedeu a cuidada análise das águas do Gerez, Aljustrel, Moura e Caldas da Rainha, e também das águas potáveis de Lisboa. Seu sucessor na regência da cadeira de Química Orgânica e analítica, Agostinho Lourenço de quem já aqui falámos (ref. 7), elaborou com Schiappa Monteiro e Tomás de Carvalho, pormenorizado relatório sobre Trabalhos Preparatorios sobre as Águas Minerais do Reino e, também na sequênçia dos trabalhos do seu antecessor, empenhou-se na análise completa das águas das termas de Chaves, Caldas de Vizela, Caldas da Rainha e outras.

Entretanto, nos princípios do ano lectivo de 1868/69 chegava a Coimbra, contratado pela Faculdade de Filosofia, o Dr. Bernard Tollens afim de dirigir os trabalhos práticos do Laboratório Chimico da Universidade. Chamado pouco depois para reger uma cadeira na Universidade de Gơttingen, o Dr. Tollens não se demorou muito tempo no Laboratório de Coimbra. Mas tanto bastou para deixar atrás de si, profundamente apaixonado pelos trabalhos práticos do Laboratório, Joaquim dos Santos e Silva, a quem fora concedido trabalhar e receber instrucçōes do ilustre químico alemão, na qualidade de simples tirocinante. Pelas qualidades e pelo interesse que então mostrou para dirigir ele próprio os trabalhos práticos do Laboratório após a partida de Tollens, foi Santos e Silva, mandado pela Universidade em Viagem de estudos à Alemanha, onde seguiu, no Laboratório da Universidade de Gottingen, de Outubro de 1871 a Agosto de 1872, os Cursos de química mineral, orgânica e aplicada dirigidos respectivamente, por Wøhler, Hübner e Tollens. De lá seguiu para Bona, onde trabalhou com Kékulé e Wallach. Regressado a Portugal em Agosto de 1873, foi investido no lugar de Chefe dos trabalhos práticos do Laboratorio Químico, no qual viria ser provido definitivamente. 
Dedicou-se então ao estudo químico de alguns derivados da cânfora (ref. 12) e realizou vários ensaios químicos sobre a essência da pimenteira falsa (ref. 13). No domínio da química hidrologica "são-lhe devidas as anályses chimicas de muitas das principaes nascentes mineraes do paiz. Começou esses estudos em 1876 por umas águas férreas da Estrada da Beira, junto a Coimbra; depois analysou sucessivamente as águas minerais das Caldas da Rainha (1876), as de Bem-Saúde (1880), as de Vidago, fonte de Campilho (1884), as da Amieira (1885), as de Piedade ou Fervença (1889 e 1894), as de Caldellas, em collaboração com o Dr. Sousa Gomes (1890), as de Marvão (1891), as de Cucos (1892), as de Alcanhoes (1893), as das Murtas, em Amarante (1894), as de Santo António de Tavira (1899), etc.".

"Occupou-se também bastante de chimica toxicológica, tendo durante 22 anos (28-2-1878 a 1899), em collaboração com alguns lentes da faculdade de Medicina (Drs. Saccadura, Lopes Vieira e Raymondo Motta) procedido às anályses toxicolbgicas ou chimico-legaes ordenadas pelos juizes da comarca de Coimbra. Escreveu uma nota sobre o movimento d'essas anályses nos laboratórios de Coimbra (Coimbra Médica, de 1900, p. 178-182) e um artigo referente ao hydrogénio sulfurado nas investigaçðes chimico-legaes.

"Em 1877, por solicitação do snr. Dr. Júlio Henriques, fez a anályse qualitativa e quantitativa dos alcalóides d'algumas quinas cultivadas em S. Thomé, da espécie succirubra, fixando o quantum de quinina em $47,56 \mathrm{~g}$ por kilogr. de quina.

"Quando, pela lei de 17 de Agosto de 1899, regulamentada pelo decreto de 16 de Novembro do mesmo anno, foram reformados os serviços médico-legaes entre nós, e creados os logares de chimicos analystas dos conselhos médico-legaes, foi indicado o nome do Snr. Santos e Silva para desempenhar o cargo de chimico analysta na circumscripção de Coimbra" (ref. 15).

Em 1874, publicou os seus Elementos de Anályse Chimica Qualitativa com o objectivo de "apresentar aos alunos que frequentam o Laboratório Chimico da Universidade de Coimbra, um quadro resumido das reacçðes principaes dos corpos que mais frequentemente se encontram, e da marcha systemática que convém seguir quando se tracta de chegar ao conhecimento da presença ou da ausência d'um qualquer d'estes corpos.

"Convencidos de que os tratados completos de anályse chimica são menos próprios para guiar os alumnos, que apenas começam neste laborioso estudo, coordenamos os apontamentos que tínhamos colligido para nosso uso particular; e, crendo prestar com elles alguns serviços ao ensino práctico da anályse chimica, ahi os apresentamos aos alumnos para os guiar nos seus primeiros estudos" (ref. 14).

Santos e Silva, "com este seu bom livro nacionalisou, digamos assim, o ensino da chimica analytica entre nós, escrevendo o primeiro guia de anályse chimica, urdido sob a base segura da experiência e da prática de laboratório" (ref. 15).

Toda esta vasta obra de Santos e Silva no domínio da análise química encontraria, entrados já no século XX, em Sousa Gomes, Álvaro Basto e demais Professores do Laboratório Químico da Unidade de Coimbra que se lhe seguiram, dignos continuadores. E, como o proprio o testemunha, foi com Santos e Silva que "aprendeu praticamente a anályse chimica" (ref. 16) esse outro grande analista químico português, nos fins do séc. XIX, que foi A. J. Ferreira da Silva, figura central da História da Análise Química no Porto. Tudo quanto neste domínio "foi realizado antes dele, no Porto, não é significativo. Mesmo os seus colaboradores e os seus sucessores imediatos se apagam perante (ele)" (ref. 1). Para além do muito mais, fundador da Sociedade de Química Portuguesa e da Revista de Química Pura e Applicada, a obra de Ferreira da Silva não se esgota de modo algum no quanto fez como químico analista. Todavia, porque esta foi a faceta da sua actividade que serviu de base à Comunicação do Prof. Dr. João Oliveira Cabral no Colóquio a que se reporta a presente nota, apenas a ela nos referiremos agora.

Ferreira da Silva sucedeu, em 1877, a Ferreira Girão na regência da cadeira de Química da Academia Politécnica do Porto. Logo em 1879, propôs e conseguiu ver aprovado um curso gratuito de química prática, na realidade um curso de análise química. No ano seguinte, é-lhe confiado pela Câmara Municipal do Porto o estudo químico das águas do rio Sousa que ultima em 1881 com um notável Relatório sobre a água do Rio Sousa e os mananciais e fontes da cidade do Porto (Porto, 1881). Neste Relatório refere as análises qualitativas e quantitativas de amostras de água daquele rio, colhidas em locais onde já não chegavam as marés, comparando os resultados com os de trinta e sete fontes do Porto, particularmente quanto à dureza. De notar que alguns dos métodos qualitativos e quantitativos que o Relatório menciona ainda hoje são empregados, embora a terminologia actual seja bastante diferente (ref. 1).

Em 1882, é encarregado da instalação do Laboratório Municpal de Química, criado pela iniciativa do então presidente do Município, Dr. Correia de Barros, sendo nomeado seu Director, no ano seguinte. Foi neste Laboratório que preparou e realizou todos os seus numerosos e importantes trabalhos de análise química bromatológica, sanitária, hidrológica, toxicologica, comercial e agrícola.

A reorganização dos estudos da Academia Politécnica do Porto, em 1885, com a criação de uma cadeira de Química Orgânica e Analítica, confiada a Ferreira da Silva, e a criação de um curso de Análise Química Comercial destinado aos alunos que seguiam o Curso Superior de Comércio, daria novos incentivos a todo aquele trabalho, sendo ponto de partida dos tratados que neste domínio publicaria, alguns anos depois: $R u$ dimentos de Química Analítica (Porto, 1893); Primeiros Elementos de Química Analítica, $1 .^{\circ}$ Vol.: - Análise Qualitativa (Porto, 1895); e Primeiros Elementos de Análise Química Quantitativa (Porto, 1897). De qualquer destes tratados se fizeram várias ediçð̄es, corrigidas, revistas e ampliadas, por vezes com alteração dos próprios títulos.

No domínio hidrológico, Ferreira da Silva apresenta, em 1896, a sua Memória e estudo químico sobre as águas minerais e potáveis de Moledo, à qual se seguiria uma intensa pesquisa química hidromineral com a análise completa das águas de Entre-os-Rios (Torre), Monção, Caldas da Saúde, Vidago, Marco de Canavezes, Valadares, Moura, Sabroso, Unhais da Serra e outras. No domínio toxicológico, são particularmente célebres as suas longas, minuciosas e perseverantes pesquisas para identificar com segurança a morfina, a narceína e a delfina que o levaram à descoberta de reacçōes novas para a eserina e a cocaína, e ainda a preciosa contribuição para a detecção e caracterização de alcalóides. Desenvolveu-se todo este trabalho no desenrolar do caso médico-legal Urbino de Freitas, um caso que envolvia fortes suspeitas de envenenamento, cujo esclareci- 
mento requeria métodos analíticos de verdadeira 'micro-análise'.

A chamada reacção Ferreira da Silva para a cocaína é descrita pelo autor no vol. I dos seus Primeiros Elementos de Química Analítica nestes termos: evapora-se uma solução que contenha cocaína, á secura, em presença de ácido nítrico fumante $(\mathrm{d}=1,4)$; " aquecido o resíduo com $\mathrm{KOH}$ em sol. alcoblico, ou somente submetido a este último tratamento, emitte cheiro privativo, que lembra o da hortelá-pimenta, e é devido ao benzoato de ethylo ou de éter benzóico (BÉHAL)". Por sua vez, a reacção Ferreira da Silva para a eserina é descrita em estoutros termos: "dissolvendo um pequeno crystal de eserina, em cápsula de porcellana, com uma gotta de $\mathrm{AZO}^{3} \mathrm{H}$ concentrado, obtem-se soluto amarello-desmaiado que, pelo aquecimento $\mathrm{a} b / \mathrm{m}$, passa sucessivamente a amarello mais carregado, e depois a alaranjado; evaporando até à secura, agitando sempre a mistura com vareta de vidro até à eliminação do líquido, observa-se, um ou dois minutos depois da exsicação, a mudança de cor do resíduo, que era amarello, para verde puro".

$\mathrm{Na}$ detecção e caracterização de alcalbides, usou ele selenito de amónio dissolvido em ácido sulfúrico concentrado, numa mistura que o Catálogo da E. Merck Produits Chimiques designa, desde 1896, por reagente Ferreira da Silva e que o próprio autor designou por reagente de Lafon e Ferreira da Silva por dele se ter servido com base nos trabalhos de Lafon sobre alcalbides. Finalmente, no dominio comercial e agrícola, foram notáveis os trabalhos de Ferreira da Silva relacionados, sob o ponto de vista da respectiva composição química, com a fiscalização de leite, cervejas saliciladas e, sobretudo, vinhos de pasto, com destaque para um lúcido relatório sobre a composição enológica regional. Nos finais do século XIX, começou a ser prática corrente adicionar-se ácido salicílico ao vinho, particularmente no caso de exportação para certos países, com o objectivo de evitar a sua deterioração durante o tempo necessário para o transporte. Não tardou, porém, que essa prática fosse condenada por se considerar que o ácido salicílico era nocivo para a saúde. O teor em ácido salicílico dos vinhos começou então a ser rigorosamente controlado, quer para evitar, quer para punir adiçð̄es fraudulentas.

Ao dobrar do século, a grande maioria dos vinhos portugueses exportados para o Brasil analisados no Laboratório Central de Análises do Rio de Janeiro, foi considerada pelo seu Director, Dr. Borges da Costa, como vinhos adulterados por aquele ácido, tendo a sua rejeição sido ordenada de imediato. Alarmado perante o labéu que esta atitude envolvia, o comércio exportador de vinhos portugueses consultou o Professor Ferreira da Silva, enviando-lhe algumas amostras para análise. Em numerosos ensaios e estudos com esses vinhos e vinhos de procedência autêntica e garantida das mesmas regiōes, demonstrou ele que a pequeníssima quantidade de ácido salicílico encontrada nos vinhos exportados era natural e não havia sido adicionada fraudulentamente. Na sua análise, fez um estudo comparativo entre os métodos de análise de vinhos salicilados propostos por Yvon (1877) e por Pellet, Grobert e Baudrimont (1881), distinguindo cuidadosamente entre sensibilidade e exactidão de uns e outros, apercebendo-se de que a primeira pode muitas vezes ser alcançada à custa da segunda e de que a sensibilidade excessiva de um método de identificação poderá induzir em erro.

Comunicados os seus resultados à Sociedade Química de França e à Academia de Ciências de Paris, as conclusøes que deles tirava seriam reconhecidas como inteiramente válidas. O método de análise usado no Laboratório de Análises do Rio de Janeiro era o método proposto por Pellet, modificado no sentido de exagerar a sua sensibilidade, donde resultava considerarem-se como levemente salicilados vinhos naturais que tinham vestígios do respectivo ácido. O próprio Henri Pellet, responsável pelo método de análise usado naquele Laboratório, reconheceria publicamente a validade das conclusð̋es de Ferreira da Silva sobre o assunto e, em particular, no tocante às críticas que fazia ao método de análise em causa.

A análise efectuada por Ferreira da Silva e a interpretação exacta dos resultados que obtivera, evitaram grave desdouro ao comércio exportador dos vinhos portugueses, defendendo seus legítimos interesses, seu crédito e a pureza de seus produtos. E foram o ponto de partida de muitos outros estudos analíticos sobre vinhos portugueses a que então se procedeu no Laboratório Municipal de Química, na cidade do Porto.

A grande actividade no domínio da análise química, em Portugal, no século XIX, particularmente aquela que no último quartel do século se desenvolveu no Laboratório Químico da Universidade de Coimbra com Joaquim dos Santos e Silva, e no Laboratorio Municipal de Química do Porto com António Joaquim Ferreira da Silva continuou-se a acção igualmente notável pelo nosso século adentro, com a particularidade de grande parte do serviço analítico que comporta ter sido desviada, em 1915, para Lisboa, e centralizada no Instituto Superior Técnico, em detrimento daqueles Laboratórios de Coimbra e Porto. Este é já, todavia, acontecimento do âmbito da História e Desenvolvimento da Ciência em Portugal no século XX. O Coloquio realizado na Academia de Ciências de Lisboa em Abril de 1985 a que nos temos vindo a referir, rotulado de I Coloquio, impôs-se programaticamente, como limite a História e Desenvolvimento da Ciência em Portugal até ao século XX. Em conformidade com essa imposição programática aqui nos detemos.

E aqui mesmo deixamos expresso o desiderato de muito em breve podermos assistir à realização de outros Coloquios que continuem a obra por aquele com muito mérito começada.

\section{BIBLIOGRAFIA}

1 - J. Oliveira Cabral, Historia da Análise Química no Porto, Sessão de 16 de Abril de 1985.

2 - C. Gerhartdt et G. Chancel, Précis d'Analyse Chimique Qualitative, Victor Masson et Fils, Paris, 1862, 2." Ediçåo.

3 - C. Barreswill et A. Sobrero, Appendice à Tous les Traités d'Analyse Chimique, Fortin, Masson et Cie, Lib., Paris, 1843. 4 - Thomé Rodrigues Sobral, Reflexठes Geraes sobre as Difficuldades de uma boa Analyse, Jornal de Coimbra, vol. VII (1814), Part. I, pp. 251-266.

5 - Thomé Rodrigues Sobral, Noticias de Differentes Minas Metálicas e Salinas, Jornal de Coimbra, vol. Vii (1814), Part. I, pp. 91-96; idem, vol. IX (1816), Part. I, pp. 221-240.

6 - Thomé Rodrigues Sobral, Ensaio Chimico da planta chamada no Brasil Mil-Homens, Jornal de Coimbra, vol. VII (1814), part. I, pp. 149-198.

7 - A.M. Amorim da Costa, A Química no I Colóquio sobre a História e Desenvolvimento da Ciência em Portugal - Parte III: Alcalóides e Polimeros, Bol. Soc. Quím., n. ${ }^{\circ} 23$ (Série II), Março 1986. pp. 37-39.

8 - Refere-se o autor à obra Analyze das Agoas Hepathizadas Marciaes do Lugar de Falla da autoria de D. Francisco de Almedia Beja e Noronha, editada pela Real Offic. da Universidade, Coimbra, 1789.

9 - L.S.M. de Albuquerque, Curso Elementar de Physica e de Chymica, Typ. Antonio Rodrigues Galhardo, Lisboa, 1824. Esta 